\title{
Distinguishing Little-Higgs Product and Simple Group models at the LHC and ILC
}

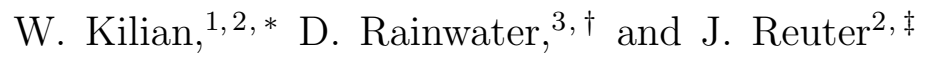 \\ ${ }^{1}$ Fachbereich Physik, University of Siegen, D-57068 Siegen, Germany \\ ${ }^{2}$ Deutsches Elektronen-Synchrotron DESY, D-22603 Hamburg, Germany \\ ${ }^{3}$ Department of Physics and Astronomy, \\ University of Rochester, Rochester, NY 14627, USA
}

(Dated: March 7, 2007)

\begin{abstract}
We propose a means to discriminate between the two basic variants of Little Higgs models, the Product Group and Simple Group models, at the next generation of colliders. It relies on a special coupling of light pseudoscalar particles present in Little Higgs models, the pseudo-axions, to the $Z$ and the Higgs boson, which is present only in Simple Group models. We discuss the collider phenomenology of the pseudo-axion in the presence of such a coupling at the LHC, where resonant production and decay of either the Higgs or the pseudo-axion induced by that coupling can be observed for much of parameter space. The full allowed range of parameters, including regions where the observability is limited at the LHC, is covered by a future ILC, where double scalar production would be a golden channel to look for.
\end{abstract}

\footnotetext{
*Electronic address: wolfgang.kilian@desy.de

${ }^{\dagger}$ Electronic address: rain@pas.rochester.edu

${ }^{\ddagger}$ Electronic address: juergen.reuter@desy.de
} 


\section{INTRODUCTION}

Little Higgs models [1] have been proposed as a solution to the hierarchy problem, the vast difference between the scales of electroweak symmetry breaking and the scale where quantum effects of gravity can no longer be neglected. The Higgs boson emerges as a pseudo-Goldstone boson of spontaneously broken chiral symmetries of a strongly interacting theory in the multi-TeV regime. The quadratically divergent contributions to the Higgs mass are canceled - unlike in supersymmetry - by particles of like statistics, chiral multiplet partners of the Standard Model (SM) fermions. The collective breaking of these symmetries generates a quadratically-divergent correction to the Higgs mass only at two-loop order, such that the models remain weakly interacting at the $\mathrm{TeV}$ scale, thereby satisfying electroweak precision data constraints.

Ideas about possible UV completions exist [2, 3, 4], but we ignore them in this paper and take Little Higgs models as low-energy effective theories. Various realizations of the Little Higgs symmetry structure have been proposed [5, 6, 7, 8, 9], grouped quite generally into two classes (cf. e.g. [10, 11]). The original models are mostly Product Group Models, where the electroweak gauge group is extended to a product gauge group that contains $S U(2)$ subgroups in at least two distinct factors. The physical $W$ and $Z$ bosons are mixtures of the gauge bosons from both factors. The global symmetry group is a simple group that includes the full gauge structure [e.g., $S U(5) \supset S U(2) \times S U(2) \times U(1)$ ], and the Higgs is part of an irreducible multiplet of pseudo-Goldstone bosons. In Simple Group Models, the electroweak $S U(2)$ gauge group is embedded in a simple (e.g., $S U(N)$ ) gauge group, while the global symmetry group has a product structure, and the Higgs is a mixture of the components of several (at least two) independent irreducible representations of the factor groups.

Although these models remain weakly interacting at the $\mathrm{TeV}$ scale, nevertheless electroweak precision observables pose constraints on their parameter space [12, 13, 14, 15] ${ }^{1}$. The decay constant $F$ of the Pseudo-Goldstone bosons which sets the mass scale for the additional vector bosons, scalars and fermions in Little Higgs models, has to lie in the range $\sim 1-4 \mathrm{TeV}$, where the upper bound comes from naturalness considerations. Usually the masses of the additional particles lie in the range of several hundred $\mathrm{GeV}$ up to a few $\mathrm{TeV}$, and are therefore (partly) directly accessible at the LHC (for an overview about Little Higgs phenomenology, cf. Ref. [18]). The proof of the Little Higgs mechanism within these models relies on the discovery of the nonlinear Goldstone boson structure of the Higgs and the cancellation mechanism of the quadratic divergences based on measurements of the couplings. This will not be possible in all cases at the LHC, but should be at a future international $e^{+} e^{-}$collider (ILC). An ILC would in any case be necessary for precision measurements. To reveal a special group theoretical realization of Little Higgs models is also quite difficult and relies on precise branching ratio measurements for the Higgs, the extra gauge bosons and fermionic states. A method to distinguish between Simple and Product Group Models, maybe even already at the LHC, would be highly welcome.

We propose a method based on the discovery and the properties of light pseudoscalar states in the spectrum, the Little Higgs pseudo-axions [19]. We first recall their properties, then show that a specific pseudo-axion coupling, to the Higgs and the $Z$ bosons, can occur only in Simple Group Models. It could thus be used as a discriminator between the two Little Higgs model classes. We then discuss relevant phenomenology at the LHC and ILC.

\footnotetext{
$\overline{1}$ There are significant corrections to mass terms and couplings in the Littlest Higgs model 16,17 .
} 


\section{A DISCRIMINATOR FOR SIMPLE AND PRODUCT GROUP MODELS}

In Little Higgs models, there can occur spontaneously broken (approximate) global $U(1)$ symmetries, corresponding to diagonal generators of the overall non-abelian global symmetry group [19]. To each of these $U(1)$ factors, which might well be anomalous, corresponds a (pseudo-) Goldstone boson which couples to fermions like a pseudoscalar, analogous to the $\eta^{(\prime)}$ meson in chiral symmetry breaking of QCD. Such a particle is an electroweak singlet, which gets a mass through explicit symmetry breaking terms and the Coleman-Weinberg potential. All couplings to SM particles are therefore suppressed by the ratio of the electroweak and the Little Higgs scale, $v / F$. All couplings to SM gauge bosons are induced by anomalous triangle loops. As a typical example for these particles, we show the situation for the so-called Simplest Little Higgs or $\mu$-model [9] in Fig. 1. Since this is a Simple Group Model, there are two multiplets of Goldstone bosons connected by a mixing angle $\tan \beta$ as in two-Higgs-doublet models. Furthermore, there is an explicit breaking of the global Little Higgs symmetries, analogous to the $\mu$ term in the MSSM. Pseudo-Axion phenomenology at the LHC and future ILC and photon colliders was discussed in [19]. There it was shown that in the Simplest Little Higgs there is a tree-level coupling $Z-H-\eta$ of the pseudo-axion to the Higgs and the $Z$, which can only arise by electroweak symmetry breaking, and is enhanced by $\tan \beta$. On the other hand, it was shown that this coupling is absent in the simplest candidate of the Product Group Models, the Littlest Higgs.

We now show that the existence of such a $Z-H-\eta$ coupling is a property of Simple Group models, and that it cannot appear in Product Group models. Hence, it serves as a discriminator between the two categories. The crucial observation is that the matrixrepresentation embedding of the two non-Abelian $S U(2)$ gauge groups, and especially of the two $U(1)$ factors within the irreducible multiplet of the pseudo-Goldstone bosons of one simple group (e.g. $S U(5)$ in the Littlest Higgs), is responsible for the non-existence of this coupling in Product Group models. It is exactly the mechanism which cancels the quadratic one-loop divergences between the electroweak and heavy $S U(2)$ gauge bosons which cancels this coupling. In Simple Group models the Higgs mass term cancellation is taken over by enlarging $S U(2)$ to $S U(N)$, and the enlarged non-Abelian rank structure cancels the quadratic divergences in the gauge sector - but no longer forbids the $Z-H-\eta$ coupling.

To leading order in the pseudo-axion field $\eta$, the parameterization of the Goldstone boson manifold in the effective Lagrangian does not depend on the basis choice of the broken generators [20]. Therefore one can take it proportional to the unit matrix, i.e. factor it out from the matrix of pseudo-Goldstone boson. This corresponds to a separation of the special $U(1)_{\eta}$ group (cf. also the discussion in Refs. [9] and [19]). Here we use $\xi=\exp [i \eta / F]$ for the pseudo-axion field and $\Sigma=\exp [i \Pi / F]$ for the non-linear representation of the remaining Goldstone multiplet $\Pi$ of Higgs and other heavy scalars. Then, for Product Group Models, the kinetic term may be expanded as

$$
\begin{aligned}
\mathcal{L}_{\text {kin. }} \sim F^{2} \operatorname{Tr}\left[\left(D^{\mu}(\xi \Sigma)^{\dagger}\left(D_{\mu}(\xi \Sigma)\right)\right]\right. & =\ldots+F^{2}\left(\partial_{\mu} \xi\right) \xi^{\dagger} \operatorname{Tr}\left[\left(D^{\mu} \Sigma\right)^{\dagger} \Sigma\right]+\text { h.c. } \\
& =\ldots-2 F\left(\partial_{\mu} \eta\right) \operatorname{Im} \operatorname{Tr}\left[\left(D^{\mu} \Sigma\right)^{\dagger} \Sigma\right]+O\left(\eta^{2}\right),
\end{aligned}
$$

where we write only the term with one derivative acting on $\xi$ and one derivative acting on $\Sigma$. This term, if nonzero, is the only one that can yield a $Z-H-\eta$ coupling.

We now use the special structure of the covariant derivatives in Product Group Models, which is the key to the Little Higgs mechanism:

$$
D_{\mu} \Sigma=\partial_{\mu} \Sigma+A_{1, \mu}^{a}\left(T_{1}^{a} \Sigma+\Sigma\left(T_{1}^{a}\right)^{T}\right)+A_{2, \mu}^{a}\left(T_{2}^{a} \Sigma+\Sigma\left(T_{2}^{a}\right)^{T}\right),
$$


where $T_{i}^{a}, i=1,2$ are the generators of the two independent $S U(2)$ groups (extra gauge structure does not matter), and $A_{i, \mu}^{a}=W_{\mu}^{a}+$ heavy fields in a suitable normalization (cf. Ref. [14]). Neglecting the heavy gauge fields and extracting the electroweak gauge bosons, we have

$$
\begin{aligned}
\operatorname{Tr}\left[\left(D^{\mu} \Sigma\right)^{\dagger} \Sigma\right] \sim & W_{\mu}^{a} \operatorname{Tr}\left[\left(\Sigma^{\dagger}\left(T_{1}^{a}+T_{2}^{a}\right) \Sigma+\left(T_{1}^{a}+T_{2}^{a}\right)^{*}\right]\right. \\
& =W_{\mu}^{a} \operatorname{Tr}\left[\left(T_{1}^{a}+T_{2}^{a}\right)+\left(T_{1}^{a}+T_{2}^{a}\right)^{*}\right]=0 .
\end{aligned}
$$

This vanishes due to the zero trace of $S U(2)$ generators. The same is true when we include additional $U(1)$ gauge group generators such as hypercharge, since their embedding in the global simple group forces them to be traceless as well. We conclude that the coefficient of the $Z-H-\eta$ coupling vanishes to all orders in the $1 / F$ expansion.

Next, we consider the kinetic term for Simple Group Models, where we use the following notation for the nonlinear sigma fields: $\Phi \zeta$, where $\Phi=\exp [i \Sigma / F]$ and $\zeta=(0, \ldots 0, F)^{T}$ is the vacuum expectation vector directing in the $N$ direction for an $S U(N)$ simple gauge group extension of the weak group. Thus, in Simple Group Models the result is the $N, N$ component of a matrix:

$$
\begin{aligned}
\mathcal{L}_{\text {kin. }} \sim F^{2} D^{\mu}\left(\zeta^{\dagger} \Phi^{\dagger}\right) D_{\mu}(\Phi \zeta) & =\ldots+\frac{i}{F}\left(\partial_{\mu} \eta\right) \zeta^{\dagger}\left(\Phi^{\dagger}\left(D_{\mu} \Phi\right)-\left(D_{\mu} \Phi^{\dagger}\right) \Phi\right) \zeta \\
& =\ldots+i F\left(\partial_{\mu} \eta\right)\left(\Phi^{\dagger}\left(D_{\mu} \Phi\right)-\left(D_{\mu} \Phi^{\dagger}\right) \Phi\right)_{N, N}
\end{aligned}
$$

To further evaluate this term, we separate the last row and column in the matrix representations of the Goldstone fields $\Sigma$ and gauge boson fields $\mathbb{V}_{\mu}$ :

$$
\Sigma=\left(\begin{array}{cc}
0 & h \\
h^{\dagger} & 0
\end{array}\right), \quad \quad \mathbb{V}_{\mu}=\left(\begin{array}{cc}
\mathbb{W}_{\mu} & 0 \\
0 & 0
\end{array}\right)+\text { heavy vector fields }
$$

The Higgs boson in Simple Group Models sits in the off-diagonal entries (one doublet for the Simplest LH and a pair of doublets for the Original Simple Group model), while the electroweak gauge bosons reside in the upper left corner.

With the Baker-Campbell-Hausdorff identity, one gets for the term in parentheses in Eq. (4):

$$
\begin{aligned}
\mathbb{V}_{\mu}+\frac{i}{F} & {\left[\Sigma, \mathbb{V}_{\mu}\right]-\frac{1}{2 F^{2}}\left[\Sigma,\left[\Sigma, \mathbb{V}_{\mu}\right]\right]+\ldots } \\
& =\left(\begin{array}{cc}
\mathbb{W}_{\mu} & 0 \\
0 & 0
\end{array}\right)+\frac{i}{F}\left(\begin{array}{cc}
0 & -\mathbb{W}_{\mu} h \\
h^{\dagger} \mathbb{W}_{\mu} & 0
\end{array}\right)-\frac{1}{2 F^{2}}\left(\begin{array}{cc}
h h^{\dagger} \mathbb{W}+\mathbb{W} h h^{\dagger} & 0 \\
0 & -2 h^{\dagger} \mathbb{W} h
\end{array}\right)+\ldots
\end{aligned}
$$

The $N, N$ entry can only be nonzero from the third term on. This can be understood as follows. In the first term, the $N, N$ component cancels by the help of the multiple Goldstone multiplets present in the Simple Group Models; it would be a mixing between the $\eta$ and the Goldstone boson(s) for the $Z^{\prime}$ state(s). If the $N, N$ component of the second term were nonzero, it would induce a $Z-H-\eta$ coupling without insertion of a factor $v$. This is forbidden by electroweak symmetry. To see this, it is important to note that $W^{3} \equiv A^{3}$ in the Simple Group Models while the hypercharge boson $B$ is a mixture of the diagonal $U(1)$ generator and the left-over diagonal generators in $S U(N)$ ( $T_{8}$ in $S U(3), T_{12}, T_{15}$ in $S U(4)$, etc.). The embedding of the Standard Model gauge group always works in such a way that hypercharge 
is a linear combination of the $T_{N^{2}-1}$ and $U(1)$ generators. This has the effect of canceling the $\gamma$ and $Z$ from the diagonal elements beyond the first two positions, and preventing the diagonal part of $\mathbb{W}_{\mu}$ from being proportional to $\tau^{3}$. By using Eq. (15) one easily sees that $\left[\Sigma, \mathbb{V}_{\mu}\right]_{N, N}$ is zero.

The third term in the expansion yields a contribution to the $Z-H-\eta$ coupling,

$$
\left(\partial^{\mu} \eta\right) h^{\dagger} \mathbb{W}_{\mu} h \sim v H Z_{\mu} \partial^{\mu} \eta
$$

Since in Simple Group models the nonlinear Goldstone boson multiplets always come in pairs, there may still be a cancellation between parameters that makes the prefactor vanish; however, this occurs only for degenerate parameter sets. By contrast, in Product Group models, the absence of this coupling is a property of the symmetry structure and occurs for all possible parameter values.

As a concrete example, we derive the $Z-H-\eta$ coupling in the Simplest Little Higgs or $\mu-$ model [9]. In this model, the EW group is enlarged to a gauged $S U(3) \times U(1)$. There are two nonlinear sigma fields, each of which parameterizes a coset space $U(3) / U(2)$ :

$$
\Phi_{1}=\exp [i \tan \beta \Theta]\left(\begin{array}{c}
0 \\
0 \\
F_{1}
\end{array}\right) \quad \Phi_{2}=\exp [-i \cot \beta \Theta]\left(\begin{array}{c}
0 \\
0 \\
F_{2}
\end{array}\right)
$$

where

$$
\Theta=\frac{1}{F}\left\{\frac{\eta}{\sqrt{2}}+\left(\begin{array}{ccc}
0 & 0 & h^{*} \\
0 & 0 & \\
h^{T} & 0
\end{array}\right)\right\}, \quad F^{2}=F_{1}^{2}+F_{2}^{2}, \quad \text { and } \quad \tan \beta=\frac{F_{2}}{F_{1}} .
$$

The gauge boson multiplet $\mathbb{V}_{\mu}$ consists of the $S U(3)_{w}$ gauge bosons and the $U(1)$ gauge boson $B_{x}$. It decomposes into the electroweak gauge bosons $W^{ \pm}, Z$, the photon $A$, and heavy vector bosons $X^{ \pm}, X^{0}, Y^{0}, Z^{\prime 0}$. In the covariant derivative acting on the Higgs multiplets, they enter via the matrix [9]

$$
\begin{aligned}
& \frac{1}{2} g A^{a} \lambda^{a}-\frac{1}{3} g_{x} B_{x}= \\
& \left(\begin{array}{ccc}
e A+\frac{g}{2 c_{w}}\left(2-3 s_{w}^{2}\right) Z+\frac{g}{2 \sqrt{3} c_{x}}\left(1-3 s_{x}^{2}\right) Z^{\prime} & \sqrt{2} W^{-} & i \sqrt{2} X^{-} \\
\sqrt{2} W^{+} & -\frac{g}{2 c_{w}} Z+\frac{g}{2 \sqrt{3} c_{x}}\left(1-3 s_{x}^{2}\right) Z^{\prime} & Y^{0}+i X^{0} \\
-i \sqrt{2} X^{+} & Y^{0}-i X^{0} & -\frac{g}{\sqrt{3} c_{x}} Z^{\prime}
\end{array}\right),
\end{aligned}
$$

which results in the following couplings of the pseudo-axion $\eta$ :

$$
\mathcal{L}_{V H \eta}=\left(\eta \stackrel{\leftrightarrow}{\partial^{\mu}} H\right)\left[\frac{m_{Z}}{\sqrt{2} F} N_{2}\left(Z_{\mu}+c_{w} c_{x} Z_{\mu}^{\prime}\right)+\frac{g}{2} N_{2} c_{\beta} s_{\beta} X_{\mu}^{0}\right]
$$

$N_{2}$ is defined as in Ref. [19]:

$$
N_{2}=\frac{F_{2}^{2}-F_{1}^{2}}{F_{1} F_{2}}=\tan \beta-\cot \beta
$$

As long as $F_{1} \neq F_{2}$, or $\tan \beta \neq 1$, there is a $Z-H-\eta$ coupling as anticipated. 
To calculate the $\eta$ production rates via gluon-gluon fusion at hadron colliders, and the $\eta$ branching ratio to observable final states, we also need its fermion couplings. In the $\mu$ model, these are as follows. The couplings to top quark and heavy top partner fermion $T$ are $g_{\eta t t}=\frac{m_{t} N_{2}}{\sqrt{2} F}-\frac{m_{t}^{2} N_{1}}{v M_{T}}$ and $g_{\eta T T}=\frac{N_{1} m_{t}}{v}$, where $N_{1}=\frac{F_{1} F_{2}}{F^{2}} \frac{\lambda_{1}^{2}-\lambda_{2}^{2}}{\lambda_{1} \lambda_{2}}$ and $\lambda_{1}, \lambda_{2}$ are the top Yukawa couplings of the model, chosen to produce the observed top quark mass and minimize $m_{T}$ (cf. Ref. [19]). The $\eta b \bar{b}$ coupling is $g_{\eta b b}=-N_{2} m_{b} / \sqrt{2} F$, and has two interesting properties: it appears only at higher orders in the expansion of $\Phi_{1,2}$ (yet remains $\mathcal{O}(v / F)$ ), and it is driven rapidly to zero the closer $F_{1}$ and $F_{2}$ are (as $\tan \beta \rightarrow 1$ ).

\section{PHENOMENOLOGY AT THE LHC}

To discuss the possible LHC $Z-H-\eta$ coupling phenomenology, we study the Simplest Little Higgs and adopt the parameter set of Ref. [9], consistent with existing EW and flavor data and the preference for a light Higgs boson. The relevant parameters are $F_{1}, F_{2}, \mu$ and $\Lambda$. For a generic scale of $\Lambda=5 \mathrm{TeV}$ at which the Little Higgs effective theory breaks down, our only free parameters are then $F_{1,2}$ and $\mu$, with the constraint that $F_{1}$ not be as small as the EW scale, $F \gtrsim 2 \mathrm{TeV}$ from EW precision constraints (primarily $\Delta T$ and four-fermion operators), and $F_{2} \gtrsim F_{1}(\tan \beta \gtrsim 1)$ to avoid too much mixing which would lead to fermion non-universality. $F_{1}=F_{2}(\tan \beta=1)$ lies right at the edge of the limits on the latter, but this point zeroes the $Z-H-\eta$ coupling and is of trivial interest. We vary $\mu$ over a slightly broader range than the calculated $m_{H}$ would suggest is allowed or favored by data.

We begin with the so called "Golden Point" discussed in Ref. [9], where

$$
F_{1}=0.5 \mathrm{TeV}, \quad F_{2}=2 \mathrm{TeV} \quad(\tan \beta=4), \quad \Lambda=5 \mathrm{TeV}
$$

which yields $m_{T}=1000 \mathrm{GeV}$ (relevant for calculating the $g g \rightarrow \eta$ rate). The Higgs and $\eta$ masses as a function of $\mu$ are shown by the $\tan \beta=4$ curves on the upper pair of and lower left panels of Fig. 11, respectively. In the lower right panel of the same figure are the $\eta$ branching ratios. For large masses, the decay to $Z H$ utterly dominates, with a very sharp transition to this regime. For most other mass values the $b \bar{b}$ decay dominates, although there is a corridor where $g g$ is large, for $\eta$ masses between 200 and $250 \mathrm{GeV}$. Also, compared to the Higgs, $\gamma \gamma$ is sizable over almost all of the parameter range. This is due to the enhanced loop factors from the pseudo-axion-top quark couplings. For more details, cf. Sec. 7.4. of Ref. [11], and Ref. [19].

There are three scenarios to consider at LHC:

1. $m_{H}>m_{\eta}+m_{Z}$ : resonant heavy Higgs production with decay to on-shell $\eta$ and $Z$. The only likely channel which could be reliably seen above QCD backgrounds and still yield reconstruction of the Higgs boson would be $\eta \rightarrow b \bar{b}$ and $Z \rightarrow \ell^{+} \ell^{-}$. Leptonic $Z$ decay would be necessary for triggering and clean identification of the $Z$ resonance. The final state is then $b \bar{b} \ell^{+} \ell^{-}(\ell=e, \mu)$.

2. $m_{\eta}>m_{H}+m_{Z}, m_{H} \lesssim 145 \mathrm{GeV}$ : resonant pseudo-axion production with decay to on-shell Higgs and $Z$. A light Higgs boson will decay primarily to $b \bar{b}$, necessitating once again leptonic $Z$ decay. Thus, the final state signature is the same as case $\# 1$, and the same analysis will apply. 
3. $m_{\eta}>m_{H}+m_{Z}, m_{H} \gtrsim 145 \mathrm{GeV}$ : as in case \#2, but the Higgs preferentially decays to a $W$ boson pair, which further decay to SM fermions. The dual-hadronic decay channel is overwhelmed by QCD background $(Z+$ jets), but the branching ratio for one leptonic and one hadronic decay is almost as large as the dual-hadronic mode, but suffers from very little QCD background. The final state is then $j j \ell^{+} \ell^{-} \ell^{ \pm}$, with a same-flavor opposite-sign pair reconstructing to the $Z$ mass.

We perform analyses to cover the two dominant final state scenarios, considering only resonant production. While there is significant parameter space where neither the $\eta$ nor the Higgs boson can be resonant, the large-QCD background environment of LHC would prohibit its observation. If Little Higgs is realized in nature and parameter space lies in this regime, only a future ILC would be able to detect this coupling. We study this in Sec. IV.
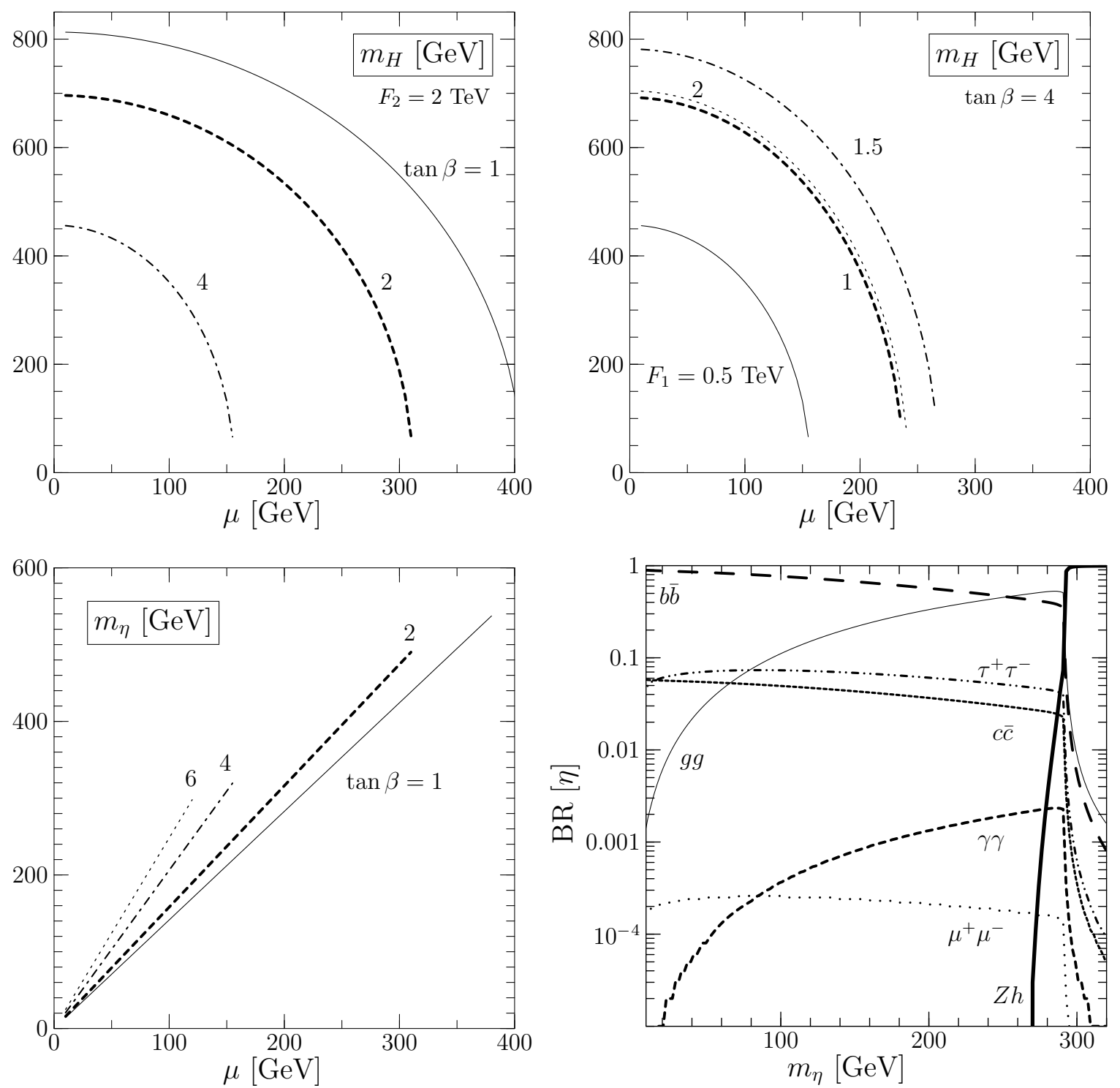

FIG. 1: Typical values for the pseudo-axion and Higgs masses in the Simplest Little Higgs model as a function of the explicit symmetry breaking parameter $\mu$. Upper pair: left, Higgs mass for fixed $F_{2}=2 \mathrm{TeV}$; right, for fixed $\tan \beta=4$. Lower pair: left, $\eta$ mass; right, $\eta$ branching fractions. 


\section{A. The $b \bar{b} \ell^{+} \ell^{-}$final state analysis}

This analysis covers both scenarios \#1 and \#2 above, where the $Z$ boson decays to an $e$ or $\mu$ pair and the daughter Higgs boson or pseudo-axion decays to $b \bar{b}$. If the Higgs boson is the daughter, it must be quite light so will likely require many tens of inverse femtobarn integrated luminosity (several years running) to discover in a standard production channel [21]. This is a fairly clean signature, as charged leptons are identifiable with extremely high efficiency and are well-measured, while the $b$ quarks give displaced vertices which are taggable by the detector, largely separating them from general QCD jet backgrounds. Except for typically small missing transverse momentum from the $b$ decays, the final state is fully reconstructible, yielding a lepton pair and the very narrow $Z$ boson peak (no dilepton continuum), a Higgs boson or pseudo-axion daughter resonance in $b$ jet pairs, and a resonance in the $b \bar{b} \ell^{+} \ell^{-}$invariant mass.

We must consider backgrounds from any process which produces the same final state. This is dominantly continuum QCD $b \bar{b} \ell^{+} \ell^{-}$production, and the small fraction of $t \bar{t}$ production which has very little missing momentum. We calculate both of these using matrix elements generated by MADGRAPH [22], which includes all spin correlations through production and decay, although both inclusive rates are known to at next-to-leading order at LHC [23, 24] and we will later apply K-factors to account for the corresponding rate enhancements.

To satisfy the basic detector requirements of observability and trigger, we impose the following kinematic cuts [25, 26]:

$$
\begin{gathered}
p_{T}(b)>25 \mathrm{GeV}, \quad|\eta(b)|<2.5 \\
p_{T}(\ell)>15 \mathrm{GeV}, \quad|\eta(\ell)|<2.5 \\
\triangle R(b b, b \ell)>0.4, \triangle R(\ell \ell)>0.2 .
\end{gathered}
$$

To identify the $Z$ peak we furthermore require that

$$
89.6<m_{\ell \ell}<92.8 \mathrm{GeV} \text {, }
$$

which corresponds to $68 \%$ capture of an on-shell $Z$ decay. To reduce the top quark background we require very little observed missing transverse momentum,

$$
\not p_{T}<30 \mathrm{GeV}
$$

To roughly simulate detector effects, we apply Gaussian smearing of the $b$ jets and charged leptons according to CMS expectations [26], as well as missing energy in $b$ jet decays according to a known distribution. This does not replace a full detector simulation, but does make our estimates more realistic, especially in terms of smeared-out invariant masses, to which we will apply a fixed window to isolate the $b \bar{b} \ell^{+} \ell^{-}$resonance peak.

Together these cuts result in a QCD $b \bar{b} \ell^{+} \ell^{-}$background of $297 \mathrm{fb}$ and a top quark background of $10.8 \mathrm{fb}$. Clearly, the QCD continuum dominates, as expected, due to the stringent missing transverse momentum restriction. The signal cross section is affected by the cuts, typically with something like a $1 / 4-1 / 3$ loss, but this varies depending on parameter choices (Higgs and pseudo-axion masses) and is not necessary to detail. As an example, for the Golden Point with $\mu=150 \mathrm{GeV}$, which results in $m_{H}=132 \mathrm{GeV}$ and $m_{\eta}=309 \mathrm{GeV}$, the cross section with cuts is $20.5 \mathrm{fb}$. For all our results we count $Z$ boson decays to both $e^{+} e^{-}$and $\mu^{+} \mu^{-}$. To illustrate the resonance feature we show in Fig. 2 the differential cross 
sections (without ID efficiencies) with respect to the visible invariant mass for signal and background, using the Golden Point parameterization and with $147<\mu<152 \mathrm{GeV}$. For these choices the pseudo-axion is the parent. Recall from Fig. 1 that $m_{\eta} \propto \mu$, while the Higgs mass is inversely proportional, but in a more complicated way that includes a plateau region in mass at low $\mu$. For $\mu=147 \mathrm{GeV}$, the Higgs mass is $158 \mathrm{GeV}$, where there is almost no branching ratio $(\mathrm{BR})$ to $b \bar{b}$, resulting in a very small rate. This is clearly in the region where one should perform instead an $j j \ell^{+} \ell^{-} \ell^{ \pm}$analysis, looking for the $H \rightarrow W^{+} W^{-}$decay; we address this channel in the next subsection.

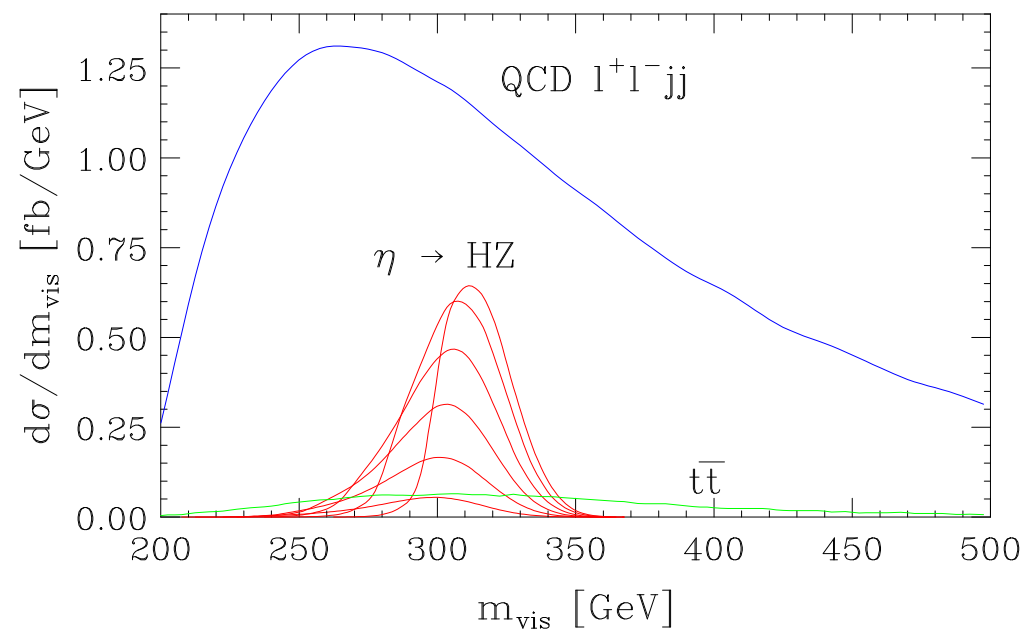

FIG. 2: Total invariant mass distribution at the LHC of the $b \bar{b} \ell^{+} \ell^{-}$final state for the Little Higgs signal (red), QCD continuum (blue) and top quark pair (green) backgrounds, using the cuts of Eqs. (14:16). Signal results are for the Golden Point with $\mu$ varying between 147 and $152 \mathrm{GeV}$.

It is possible to perform a simple "bump-hunting" analysis, although it is far from optimum. We observe significant angular correlations in the leptons and $b$ jets which can be used to reject the backgrounds further. Fig. 3 shows the two most important correlations, the $b-b$ and $\ell-\ell$ lego plot separations. There are distinct differences between resonant and continuum production. For the Golden Point and $\mu=150 \mathrm{GeV}$, it is possible to reduce the QCD background by a factor 3 while losing only $15 \%$ of the signal. This does sculpt the total invariant mass distribution somewhat, but reduces by about half the amount of luminosity required for discovery. In general, though, the angular cut choice needed changes with choice of input parameters, as it depends ultimately on the decay kinematics - how boosted the daughters are. We don't attempt such a complicated analysis here, but point out the correlations for future detailed work at the detector level.

We present our overall results then, without optimization using angular correlations, in Fig. 4 in terms of the required luminosity for one LHC detector to make a $5 \sigma$ observation of the $b \bar{b} \ell^{+} \ell^{-}$final state above QCD backgrounds. To obtain this we used ID efficiency factors of $50 \%$ for each $b$ jet and $95 \%$ for each charged lepton, and a capture efficiency of $68 \%$ for the dilepton mass window (signal only). We also include K-factors for signal and background to take into account the large QCD rate corrections: 2.3 for the signal [27], 1.3 for the QCD $Z b \bar{b}$ [23] background, and 1.4 for $t \bar{t}$ [24]. Our results are only estimates, not the optimal reach, and not comprehensive over parameter space. In addition to the Golden Point, we show two other cases for different choices of $F_{1,2}$. For $F_{1,2}=1.0,2.0 \mathrm{TeV}(\tan \beta=2)$, the collective scale $F^{2}=F_{1}^{2}+F_{2}^{2}$ is approximately the same, but the $Z-H-\eta$ coupling squared 
is reduced by an order of magnitude. For $F_{1,2}=1.0,4.0 \mathrm{TeV}(\tan \beta=4$, same as the Golden Point), the scale $F$ is about twice as large, but the coupling strength is unchanged.

For $H \rightarrow Z \eta$, shown by crosses, large $F$ values drive $m_{H}$ to several hundred GeV. The $Z \eta \mathrm{BR}$ drops rapidly with increasing Higgs mass, as it is out-competed by the $V V$ modes' double longitudinal-polarization enhancement. Thus a heavy Higgs boson decaying to $Z \eta$ would likely be observable with LHC luminosity only for fairly small values of $F_{1,2}$ and $\mu$.

The $\eta \rightarrow Z H$ case suffers a somewhat different fate, as shown by the diamonds. Typically for any given $F_{1,2}$ there is only a very small range of $\mu$ which can be addressed. If $\mu$ is too large, $m_{H}$ is smaller than the LEP limit. Only a few GeV lower than this limit, the Higgs boson mass lies in the region where decays to weak bosons dominate, thus the BR to $b \bar{b}$ drops to zero and the $j j j j \ell^{+} \ell^{-}$analysis takes over. For larger $F_{1,2}$ than at the Golden Point, $m_{\eta}$ increases, lowering the production rate, even though the $\mathrm{BR}$ to $Z H$ is still large.

\section{B. The $j j \ell^{+} \ell^{-} \ell^{ \pm}$final state analysis}

For $m_{H} \gtrsim 140 \mathrm{GeV}$, the BR to $b \bar{b}$ drops off dramatically, being replaced mostly by $W^{+} W^{-}$. This requires a different analysis. Because the $W$ boson leptonic BR is smaller than that to quarks (jets), and we already have trigger leptons in the final state from the very sharp $Z$ boson resonance, the obvious final state to consider is the largest in terms of BR: $W^{+} W^{-} \rightarrow j j j j$. It is fully reconstructible, and typically better so than the $b \bar{b} \ell^{+} \ell^{-}$ case, because light-flavor jets typically give much less missing energy. However, this channel suffers from a QCD $Z$ +jets background of several hundred fb [28]. A quick analysis of this channel shows that in principle it would be statistically possible for some parameter space, but the pseudo-axion resonance would peak at about the same places the QCD continuum does, with a $S / B$ ratio of about $1 / 50$, which makes prospects dodgy.

Instead, we investigate the $H \rightarrow W W \rightarrow \ell \nu j j$ channel, which has $2 / 3$ the $\mathrm{BR}$ of the all-hadronic channel, but about 1/100 the background, which comes almost exclusively from $Z W j j$ production [29]. The final state is then three charged leptons, two jets and missing

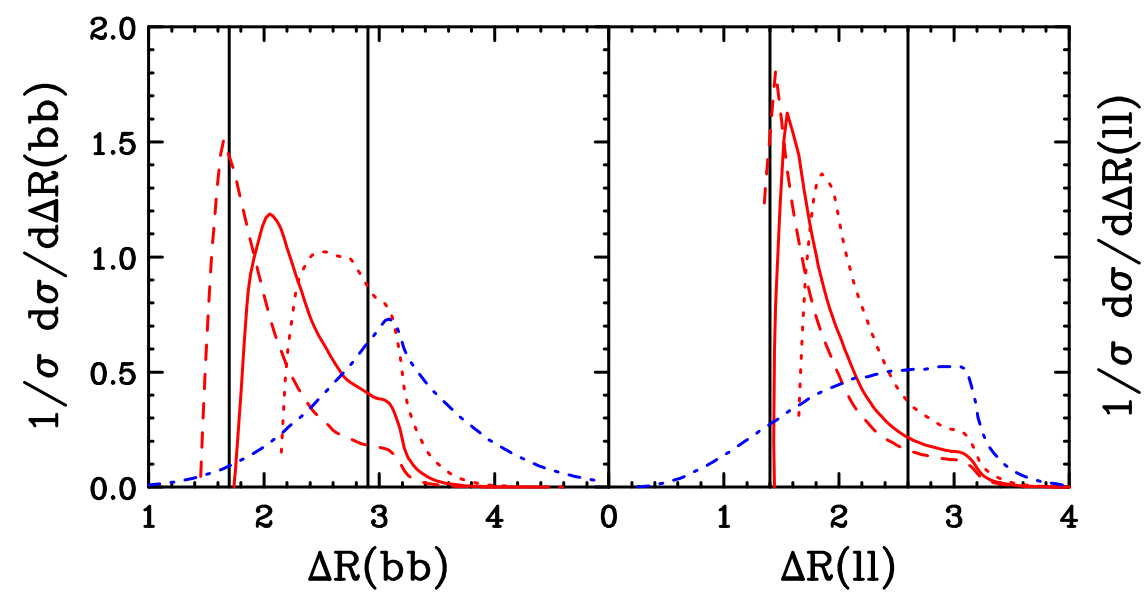

FIG. 3: Normalized angular correlations in the lego plot separation for the $b$ jet pair (left) and lepton pair (right). The signal is shown in red for the Golden point and $\mu=147,150,152 \mathrm{GeV}$ using dotted, solid and dashed lines, respectively. The QCD continuum backgrounds is shown in blue (dot-dashed). Vertical lines represent cuts used for the $\mu=150$ case as described in the text. 


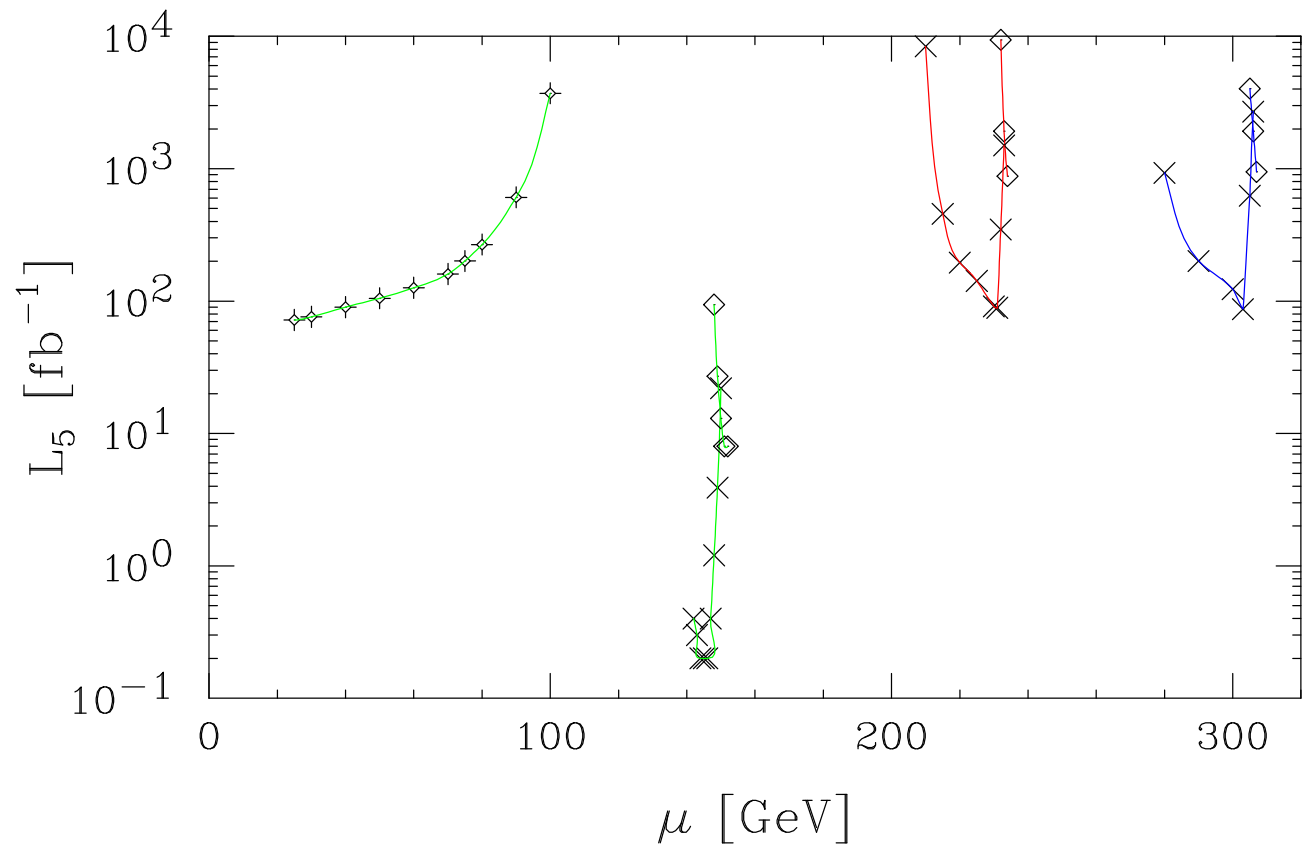

FIG. 4: Required integrated luminosity for one LHC detector to make a $5 \sigma$ observation of the Little Higgs pseudo-axion. The Golden Point is green, while blue (red) are for $F_{1,2}=1,2(1,4) \mathrm{TeV}$. Crosses represent the $b \bar{b} \ell^{+} \ell^{-}$analysis for $g g \rightarrow H \rightarrow \eta Z$, while diamonds are the same for $g g \rightarrow$ $\eta \rightarrow H Z$ (cf. Subsec. IIA), and X symbols are for $g g \rightarrow \eta \rightarrow H Z$ where the Higgs boson is heavier and decays to $j j \ell \nu$ (cf. Subsec. IIIB). Crosses for the larger $F_{1,2}$ choices are above $10^{4}$.

transverse momentum. The basic kinematics cuts for detector acceptance and trigger are:

$$
\begin{array}{r}
p_{T}(j)>20 \mathrm{GeV}, \quad|\eta(j)|<4.5, \\
p_{T}(\ell)>15 \mathrm{GeV}, \quad|\eta(\ell)|<2.5 \\
\triangle R(j j, j \ell)>0.4, \triangle R(\ell \ell)>0.2 \\
89.6<m_{\ell \ell}<92.8 \mathrm{GeV}, \quad p_{T}<30 \mathrm{GeV} .
\end{array}
$$

The $Z$-pole cut is the same as before, applied on the $Z$ decay products explicitly (combinatorics will be a very minor correction). Typically only about $25 \%$ of the signal survives, but the total continuum background is now only a factor of two larger than the signal for the Golden Point and $\mu=145 \mathrm{GeV}$.

With only one neutrino, it is straightforward to construct a transverse mass for the entire system, which peaks very close to the pseudo-axion mass as expected, with only minor smearing due to detector effects. We show examples for illustration in Fig. 5 for the Golden Point and $F_{1,2}=1.0,2.0 \mathrm{TeV}$, for a few values of $\mu$. Since $m_{\eta} \propto \mu$, larger peak values of $m_{T}$ are for larger values of $\mu$. The Golden Point would produce a signal far above the background, trivially observable, at least in the region of $142<\mu<150 \mathrm{GeV}$ where the $\eta$ can be resonant and the Higgs boson has at least a modest BR to $W W$. The $F_{1,2}=1.0,2.0 \mathrm{TeV}$ cases yield a signal of the same size as the background, also quite easy to observe but naturally requiring more statistics.

This analysis is generally powerful wherever there is a decent signal rate, due to the small background. Using the same ID efficiencies and signal K-factor as in Sec. IIIA and a generic QCD K-factor of 1.3 for the background ( $W Z j j$ is not known at NLO), we summarize our 
results again in Fig. 4 with points using an $\mathrm{X}$ symbol. The left edge of each curve is cut off by values of $\mu$ for which the $\eta$ cannot be resonant, so the rate is hopelessly small. As $\mu$ increases, $m_{\eta}$ increases and $m_{H}$ decreases, opening up more phase space for the decay, resulting in more events passing the cuts, which in turn means less luminosity required. However, below $H \rightarrow W W$ threshold the BR to $W W$ begins to falls off steeply, resulting instead in an increase in required luminosity with increasing $\mu$. At some point, the $H \rightarrow b \bar{b}$ analysis becomes more powerful, and the curves cross those with the diamond points.

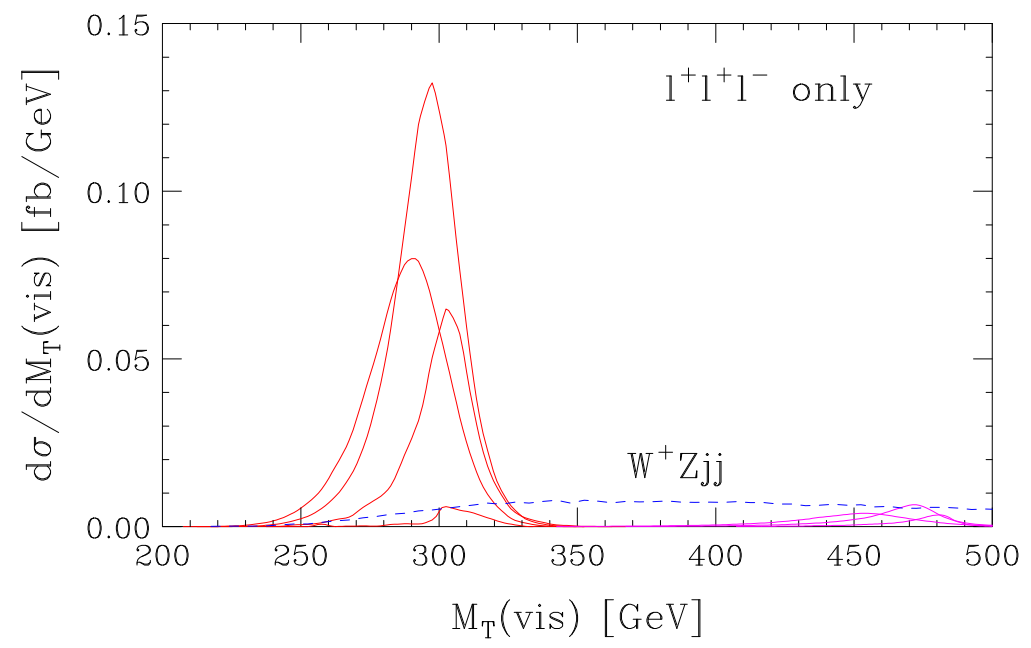

FIG. 5: Transverse mass distribution of the visible $\ell^{+} \ell^{+} \ell^{-} j j p_{T}$ system in $g g \rightarrow \eta \rightarrow Z H, H \rightarrow$ $W W$ resonant production at the LHC. Red and magenta curves are for the Golden Point and $F_{1,2}=1.0,2.0 \mathrm{TeV}$, respectively, and the dashed blue curve is the $W^{+} Z j j$ background.

\section{PHENOMENOLOGY AT AN ILC}

At a future ILC, one would not have to rely on on-shell production to prove or place a limit on the existence of a $Z-H-\eta$ coupling. As long as it is present and $H \eta$ pair production is kinematically allowed, the pseudo-axion could be seen. Fig. [6] shows the total cross section at an ILC for various $\sqrt{s}$ for this channel at the Golden Point for three different values of $\mu=$ $24.2 / 97 / 150 \mathrm{GeV}$, for which $m_{\eta}=309.2 / 200 / 50 \mathrm{GeV}$ and $m_{H}=131.7 / 368.4 / 451.3 \mathrm{GeV}$, respectively. The maximum cross section is of the order of $0.4-1.2 \mathrm{fb} \times \tan ^{2} \beta$, as shown in the left panel of Fig. 6. Since in the Simplest Little Higgs there is a destructive interference between the SM $Z$ boson and the $Z^{\prime}$ boson, the maximum total cross section in the full model goes down by roughly a quarter, as shown in the right panel.

Depending on the mass of the pseudo-axion, the main decay channel for the $\eta$ varies from (cf. Fig. (1) $b \bar{b}$ when fairly light, to two gluons (jets) for intermediate masses, and finally only $Z H$ for masses above that threshold, about $m_{\eta} \gtrsim 290 \mathrm{GeV}$ at the Golden Point. For such heavy pseudo-axions, one would look into the $Z H H$ final state. The cross section for this process is shown in the left panel of Fig. 7) (this and the other ILC simulations were performed by the BSM extensions of the event generator O'MEga/WhizARD [32, 33]). The dashed curve is the SM cross section, the dotted line shows the signal process without a $Z^{\prime}$ resonance or with one above $1.5 \mathrm{TeV}$, while the solid line is the prediction for $M_{Z^{\prime}}=1.15 \mathrm{TeV}$. For the parameter values considered here the Higgs mass is $131.7 \mathrm{GeV}$, so that the six-fermion final 

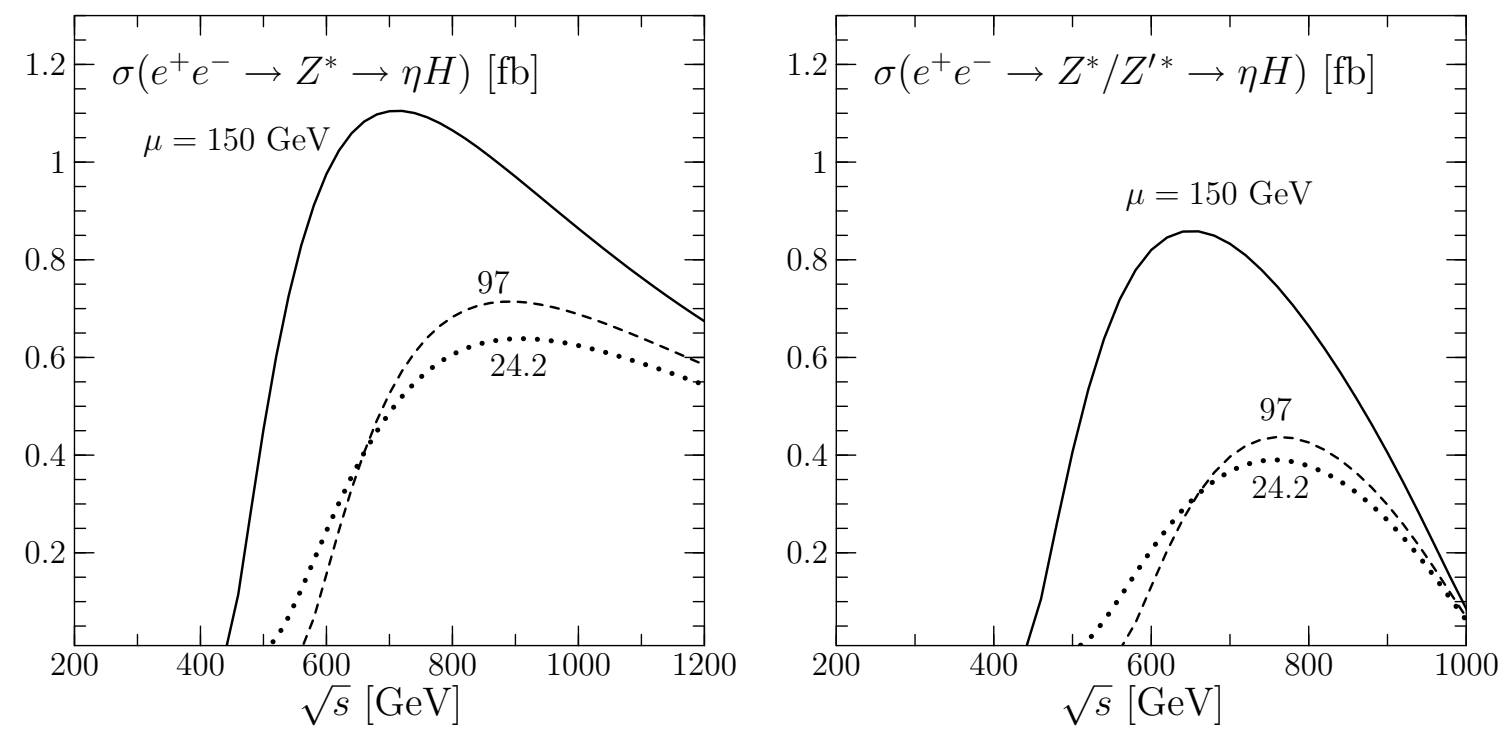

FIG. 6: Total cross section for $H \eta$ production at an ILC for different values of $\mu, m_{\eta}, m_{h}$ : the Golden Point with 150/309.2/131.7 GeV (solid), 97/200.0/368.4 GeV (dashed), and 24.2/50.0/451.3 GeV (dotted). The left panel is without the presence of a $Z^{\prime}$ resonance, while the right panel includes destructive $Z-Z^{\prime}$ interference for $m_{Z^{\prime}}=1152 \mathrm{GeV}$.
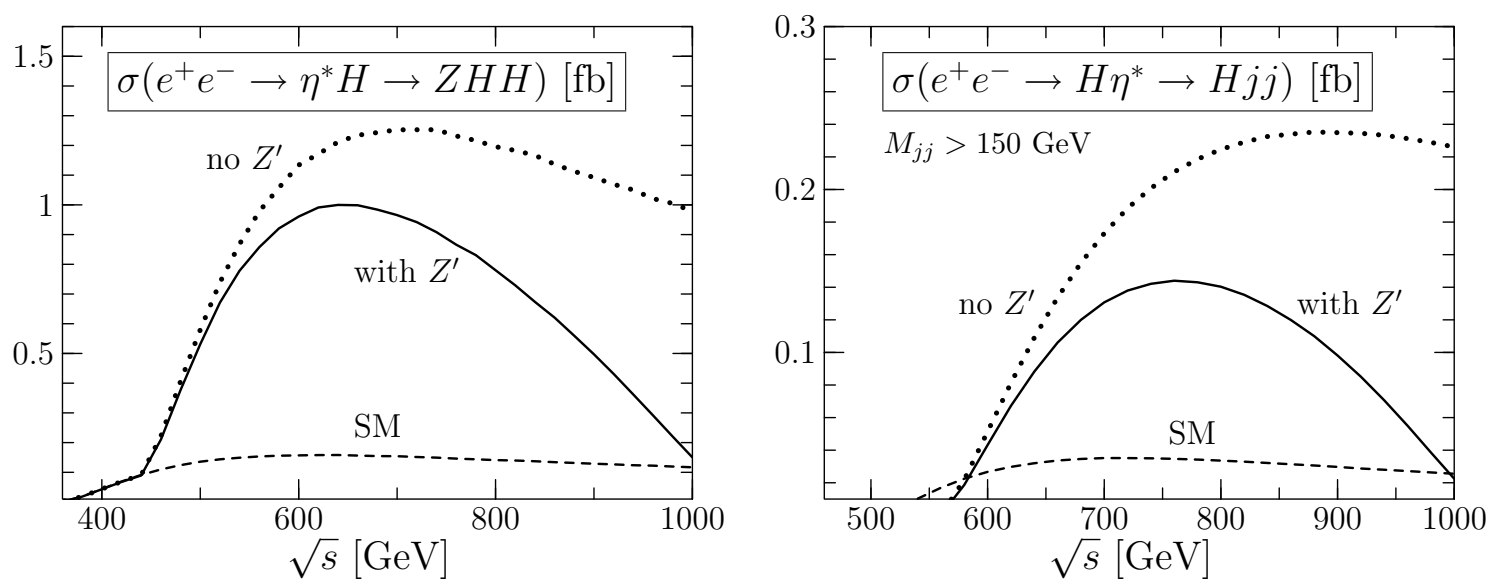

FIG. 7: Cross section in the $\mu$ model pseudo-axion with (solid line) and without (dotted line) the $Z^{\prime}$ resonance at the Golden Point. The dashed line is the SM prediction. On the left is the $Z H H$ final state for $\mu=150 \mathrm{GeV}$, while the right is the $H j j$ final state for $\mu=97 \mathrm{GeV}$.

state of interest consists of four $b$ jets and a lepton pair. The SM $e^{+} e^{-} \rightarrow Z H H$ process was extensively studied to analyze the triple Higgs coupling measurability at an ILC [30, 31]. It was found that the search limit for this process is severely statistics-limited and hence hard to analyze. Fig. 7 shows that even for the presence of a destructive interference with a $Z^{\prime}$ boson, the cross section in the Simplest Little Higgs is larger than in the SM by a factor of five, allowing for a reliable analysis of the final state. The pseudo-axion shows up as a sharp spike in the bbll spectrum, since even if the $Z H$ decay is kinematically allowed the width is of the order of a few hundred $\mathrm{MeV}$ and hence far smaller than any conceivable detector resolution. 

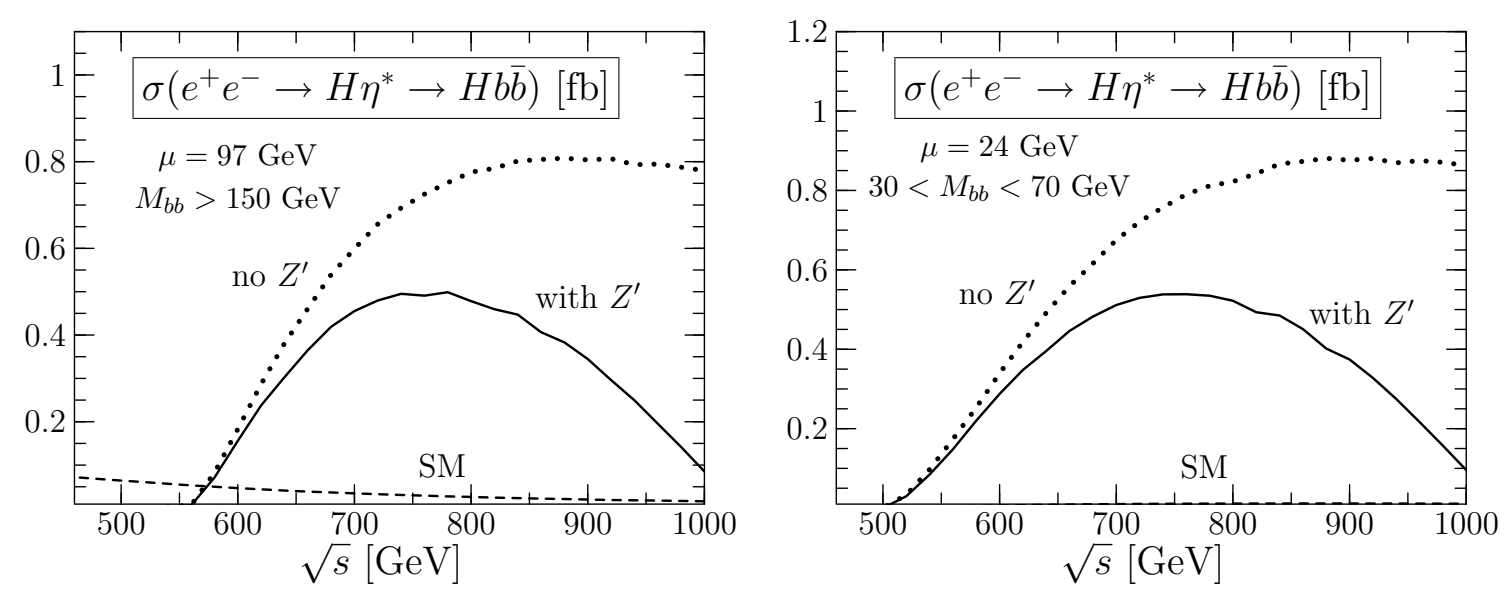

FIG. 8: Cross section for $H b \bar{b}$ production in the $\mu$-model pseudo-axion with (solid line) and without (dotted line) the $Z^{\prime}$ resonance at the Golden Point. The dashed line is the SM prediction. On the left is $\mu=97 \mathrm{GeV}$, right $\mu=24.2 \mathrm{GeV}$.

If the pseudo-axion mass lies between roughly 200 and $290 \mathrm{GeV}$, the $\eta$ has a large branching fraction into a gluon pair, of the order of $30-50 \%$, which can even become dominant close to the $Z H$ threshold. For this parameter range, the Higgs boson decays completely

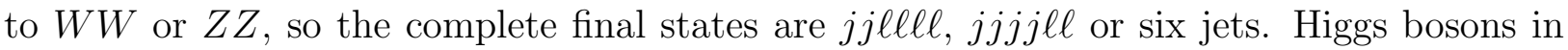
an intermediate mass range could be reconstructed at an ILC [30] fairly well, and although there would be combinatorial background in the $H \rightarrow 4 j$ channel, this would not degrade the pseudo-axion search. The right panel of Fig. 7 shows the cross section to $H j j$, where a cut on the dijet invariant mass has been applied to reduce the SM Higgsstrahlung background which is shown as a dashed line. Again, the dotted line is the prediction without a low-lying $Z^{\prime}$, while the solid line shows the destructive $Z-Z^{\prime}$ interference. For the Golden Point with $F_{2}=2 \mathrm{TeV}$ and $\tan \beta=4$, there is a critical point at $\mu \sim 128 \mathrm{GeV}$ where the pseudo-axion and Higgs boson are degenerate (at $\sim 264 \mathrm{GeV}$ ). But vector boson-mediated production cannot yield two identical particles (under the assumption of CP conservation); hence this must be either a scalar and a pseudoscalar or a scalar and a vector. From the normalization of the $b \bar{b}$ peak, which does not fit to the Higgs boson, one could deduce that on one side of the event there must be a degenerate pseudoscalar present.

The best case is where $b \bar{b}$ is the dominant $\eta$ decay mode, i.e. for pseudoscalar masses below $250 \mathrm{GeV}$. The SM background is mainly Higgsstrahlung with $Z \rightarrow b \bar{b}$ decay, which can be distinguished from the signal by applying a cut to the $b \bar{b}$ invariant mass. For the parameter space under consideration, the Higgs decays to a vector boson pair which can be measured semi-leptonically. Fig. 8 shows the cross section for the production of a Higgs boson in association with a $b \bar{b}$ pair, on the left for a pseudo-axion mass of $200 \mathrm{GeV}$, on the right for an extremely light $\eta$ with $m_{\eta}=50 \mathrm{GeV}$. For the former case one can cut out all bottom quark pairs below $150 \mathrm{GeV}$ to get rid of the $Z$ background; while this is not a very rigorous cut, it already reduces the SM background to a negligible level. To extract the light pseudoscalar we adopt a window between 30 and $70 \mathrm{GeV}$ for $m_{b b}$. Again, Fig. 8 shows as a dotted line the cross section without $Z^{\prime}$ interference, and solid with the $m_{Z^{\prime}}=1.15 \mathrm{TeV}$. In both cases a few hundred $\mathrm{fb}^{-1}$ should suffice to observe $H-\eta$ production in that channel and thus establish the presence of a $Z-H-\eta$ coupling. 


\section{CONCLUSIONS}

We have identified an important distinction between Product Group and Simple Group Little Higgs models crucially involving the pseudo-axion corresponding to ungauged diagonal generators in the former case, and the pseudoscalar combination of anomalous $U(1)$ diagonal generators in the latter. By consideration of quantum numbers alone, a coupling between the pseudo-axion, Higgs boson and $Z$ gauge boson is allowed. However, by construction such a coupling must vanish to all orders in Product (Gauge) Group models, where the global Little Higgs symmetry group is simple. In Simple Group models it is proportional to the difference between the vevs of the chiral field which occur in pairs. Observing resonant production at the LHC, either $\eta \rightarrow Z H$ or $H \rightarrow Z \eta$, or Drell-Yan $H \eta$ production in $e^{+} e^{-}$collisions, would conclusively rule out a Product Group realization of the Little Higgs mechanism were a candidate Little Higgs found at the LHC. Note, however, that this coupling would also vanish in Simple Group models with an imposed T-parity [34], as the discrete symmetry forces $F_{1}=F_{2}$.

The phenomenology at LHC is in principle straightforward, breaking down into three classes, depending on whether the pseudo-axion or Higgs boson is heavier, and whether the Higgs boson decays primarily to a pair of bottom quarks or $W$ gauge bosons. We find that observation of $g g \rightarrow \eta \rightarrow Z H \rightarrow \ell^{+} \ell^{-} b \bar{b}$ is in general possible only for a very small range in $\mu$, where the Higgs boson is light enough to decay to bottom quarks but is not yet ruled out by the LEP results. For small values of $F, 1-2 \mathrm{TeV}$, this could be achieved at LHC with a reasonable few hundred inverse femtobarn of luminosity, but for large values of $F$, a few $\mathrm{TeV}$, the $\eta$ is more massive thus harder to produce, so only the luminosity-upgraded LHC (SLHC) would have a chance of observation of this coupling. (This would furthermore probably require different cuts, such as a less restrictive $\not p_{T}$ cut in the $b \bar{b} \ell^{+} \ell^{-}$analysis, which likely would allow much more background from $t \bar{t}$.) For similar values of $F_{1}$ and $F_{2}$, the coupling is too small. If the Higgs boson is heavier, one generally loses as it preferentially decays to two longitudinal gauge bosons rather than $Z \eta$, so the $\mathrm{BR}$ drops to negligible values. If the pseudo-axion is resonant but the Higgs boson decays to gauge bosons, a multilepton analysis works extremely well, especially for $F$ around 1-2 TeV: even a few inverse femtobarn cover much of the possible parameter space. However, we find that LHC has large gaps in coverage, for regions where neither the Higgs boson nor the pseudo-axion can be resonant. This is precisely where a linear collider would be crucial.

At a future linear collider the detection of the pseudo-axion would be quite easy using the $Z-H-\eta$ coupling in scalar pair production. The cross section for the whole parameter space is large enough to give several hundred pseudo-axions for reasonable luminosity, irrespective of any of $\eta \rightarrow Z H$ or $H \rightarrow Z \eta$ being on-shell. This closes the holes in the LHC discovery range. An important point is a destructive $Z / Z^{\prime}$ interference in that channel which is most severe for the lowest $F$ scales but does not jeopardize the signal. In the case of an onshell decay $\eta \rightarrow Z H$ the $Z H H$ final state is enhanced compared to the SM by a factor of at least two for the region favored by electroweak precision data and up to an order of magnitude for large $\tan \beta$. The same holds for the $b \bar{b}$ decay mode of the pseudo-axion where the enhancement over the SM is less pronounced. But here the pseudo-axion is easily visible even for bad signal to background ratio as a sharp peak in the $b b$ invariant mass spectrum, whose width is completely given by the detector resolution. So even for mixing angles as small as $\tan \beta \sim 1.5$ the pseudo-axion coupling $Z-H-\eta$ coupling can be detected at an ILC. 


\section{Acknowledgments}

We thank Uli Baur for a critical reviews of the manuscript. This research was supported in part by the National Science Foundation under Grant No. PHY99-07949, the U.S. Department of Energy under grant No. DE-FG02-91ER40685, and by the Helmholtz-Gemeinschaft under Grant No. VH-NG-005.

[1] N. Arkani-Hamed, A. G. Cohen, H. Georgi, Phys. Lett. B 513 (2001) 232;

N. Arkani-Hamed, A. G. Cohen, T. Gregoire, and J. G. Wacker, JHEP 0208 (2002) 020.

[2] E. Katz, J. y. Lee, A. E. Nelson and D. G. E. Walker, arXiv hep-ph/0312287;

M. Piai, A. Pierce and J. Wacker, arXiv hep-ph/0405242.

[3] D. E. Kaplan, M. Schmaltz and W. Skiba, arXiv/hep-ph/0405257

[4] A. Birkedal, Z. Chacko and M. K. Gaillard, arXiv hep-ph/0404197;

P. H. Chankowski, A. Falkowski, S. Pokorski and J. Wagner, Phys. Lett. B598 (2004) 252.

[5] N. Arkani-Hamed, A. G. Cohen, E. Katz, and A. E. Nelson, JHEP 0207 (2002) 034.

[6] N. Arkani-Hamed et al., JHEP 0208 (2002) 021.

[7] D. E. Kaplan and M. Schmaltz, JHEP 0310 (2003) 039.

[8] I. Low, W. Skiba, and D. Smith, Phys. Rev. D 66 (2002) 072001;

S. Chang and J. G. Wacker, Phys. Rev. D 69 (2004) 035002;

T. Gregoire, D. R. Smith, and J. G. Wacker, Phys. Rev. D 69 (2004) 115008;

W. Skiba and J. Terning, Phys. Rev. D 68 (2003) 075001;

S. Chang, JHEP 0312 (2003) 057.

[9] M. Schmaltz, JHEP 0408 (2004) 056.

[10] T. Han, H. E. Logan and L. T. Wang, JHEP 0601, 099 (2006).

[11] S. Kraml et al., CPNSH report: the hitchhikers guide to non-standard Higgs physics, hep-ph/0608079.

[12] C. Csáki et al., Phys. Rev. D 67 (2003) 115002; Phys. Rev. D 68 (2003) 035009.

[13] J. L. Hewett, F. J. Petriello, and T. G. Rizzo, JHEP 0310 (2003) 062.

[14] W. Kilian and J. Reuter, Phys. Rev. D 70 (2004) 015004.

[15] S. C. Park and J.-H. Song, arXiv hep-ph/0306112;

M.-C. Chen and S. Dawson, Phys. Rev. D 70 (2004) 015003;

R. Casalbuoni, A. Deandrea, and M. Oertel, JHEP 0402 (2004) 032.

[16] A. J. Buras, A. Poschenrieder, S. Uhlig and W. A. Bardeen, arXiv:hep-ph/0607189.

[17] J. Boersma, arXiv hep-ph/0608239.

[18] T. Han, H. E. Logan, B. McElrath, and L.-T. Wang, Phys. Rev. D 67 (2003) 095004;

G. Burdman, M. Perelstein, and A. Pierce, Phys. Rev. Lett. 90 (2003) 241802

[Erratum-ibid. 92 (2004) 049903];

M. Perelstein, M. E. Peskin, and A. Pierce, Phys. Rev. D 69 (2004) 075002;

G. Azuelos et al., Eur. Phys. J. C 39S2, 13 (2005);

B. C. Allanach et al., hep-ph/0602198;

K. Cheung, C. S. Kim, K. Y. Lee and J. Song, hep-ph/0608259.

[19] W. Kilian, D. Rainwater and J. Reuter, Phys. Rev. D 71, 015008 (2005); hep-ph/0507081.

[20] C. G. Callan, S. R. Coleman, J. Wess and B. Zumino, Phys. Rev. 177, 2247 (1969).

[21] See e.g. K. S. Cranmer, arXiv hep-ph/0512154. 
[22] T. Stelzer, F. Long, Comput. Phys. Commun. 81 (1994) 357.

[23] J. Campbell, R. K. Ellis and D. L. Rainwater, Phys. Rev. D 68, 094021 (2003).

[24] P. Nason, S. Dawson and R. K. Ellis, Nucl. Phys. B 303, 607 (1988);

R. Bonciani, S. Catani, M. L. Mangano and P. Nason, Nucl. Phys. B 529, 424 (1998);

N. Kidonakis, E. Laenen, S. Moch and R. Vogt, Phys. Rev. D 64, 114001 (2001);

M. Beneke et al., arXiv hep-ph/0003033 (cf. Sec. 2).

[25] ATLAS TDR, report CERN/LHCC/1999-15 (1999).

[26] CMS TDR, report CERN/LHCC/2006-001 (2006).

[27] R. V. Harlander and W. B. Kilgore, Phys. Rev. Lett. 88, 201801 (2002);

R. V. Harlander and W. B. Kilgore, JHEP 0210, 017 (2002); C. Anastasiou, K. Melnikov and F. Petriello, Phys. Rev. Lett. 93, 262002 (2004);

C. Anastasiou, K. Melnikov and F. Petriello, Nucl. Phys. B 724, 197 (2005).

[28] M. L. Mangano, M. Moretti, F. Piccinini, R. Pittau and A. D. Polosa, JHEP 0307, 001 (2003).

[29] V. D. Barger, T. Han, J. Ohnemus and D. Zeppenfeld, Phys. Rev. D 41, 2782 (1990).

[30] J. A. Aguilar-Saavedra et al. [ECFA/DESY LC Physics Working Group], "TESLA Technical Design Report Part III: Physics at an e+e- Linear Collider," hep-ph/0106315.

S. Heinemeyer et al., hep-ph/0511332 (and references therein).

[31] A. Djouadi, W. Kilian, M. Mühlleitner and P. M. Zerwas, Eur. Phys. J. C 10, 27 (1999).

[32] T. Ohl, O'Mega: An Optimizing Matrix Element Generator, hep-ph/0011243, M. Moretti, T. Ohl, J. Reuter, hep-ph/0102195.

[33] W. Kilian. WHIZARD MANUAL, in *2nd ECFA/DESY Study 1998-2001* 1924-1980, LC-TOOL-2001-039, Jan 2001; W. Kilian, T. Ohl, and J. Reuter, in preparation.

[34] A. Martin, arXiv hep-ph/0602206. 